Rhode Island College

Digital Commons @ RIC

\title{
Barriers Faced by Nurses Caring For End-of-Life Patients on a Medical Surgical Unit
}

\author{
Beth Dufour
}

Follow this and additional works at: https://digitalcommons.ric.edu/etd

Part of the Perioperative, Operating Room and Surgical Nursing Commons

\section{Recommended Citation}

Dufour, Beth, "Barriers Faced by Nurses Caring For End-of-Life Patients on a Medical Surgical Unit" (2018). Master's Theses, Dissertations, Graduate Research and Major Papers Overview. 264.

https://digitalcommons.ric.edu/etd/264

This Major Paper is brought to you for free and open access by the Master's Theses, Dissertations, Graduate Research and Major Papers at Digital Commons @ RIC. It has been accepted for inclusion in Master's Theses, Dissertations, Graduate Research and Major Papers Overview by an authorized administrator of Digital Commons @ RIC. For more information, please contact digitalcommons@ric.edu. 


\section{BARRIERS FACED BY NURSES CARING FOR END-OF-LIFE PATIENTS ON A MEDICAL SURGICAL UNIT}

by

\section{Beth Dufour}

A Major Paper Submitted in Partial Fulfillment

of the Requirements for the Degree of

Master of Science in Nursing

in

The School of Nursing

Rhode Island College

2018 


\begin{abstract}
Many patients will spend their final days and die in the hospital. The hospital setting provides unique challenges to providing high quality end of life care. The nurses' role in caring for end-of-life patients in this challenging setting is extremely important. The purpose of this study was to determine what barriers registered nurses (RNs) face while caring for end-of-life patients on a medical-surgical unit in the hospital setting. A survey was used to identify these barriers on a medical surgical unit at Westerly Hospital. The theory of Nurse Moral Distress by Corley (2002) was used to guide this study. The results of the survey reinforced that nurses in the medical-surgical setting are faced with barriers that make caring for end-of-life care patients challenging. The most frequent barriers identified to providing good end-of-life care were a lack of time and education/training. Nurses described "managing your patient assignment is difficult" while caring for an end-of-life patient, especially because their "is never enough time". These results are useful in identifying areas in which the Advanced Practice Nurse can help influence change in this setting such as leading educational initiatives and creating and implementing policies and procedures. Through these efforts, the APRN can work with nurses and the interdisciplinary team to improve patient and family experiences during this difficult time.
\end{abstract}




\section{Table of Contents}

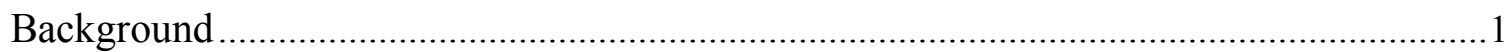

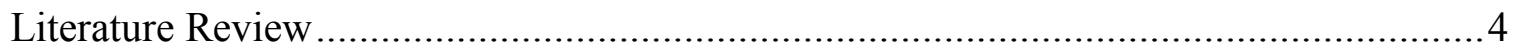

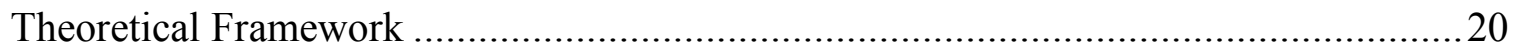

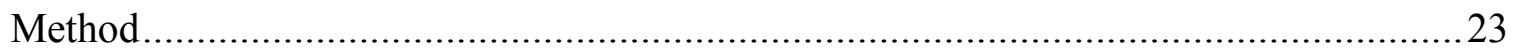

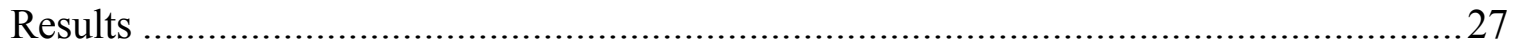

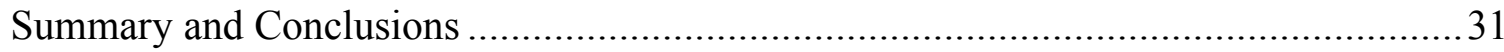

Recommendations and Implications for Advanced Nursing Practice............................... 34

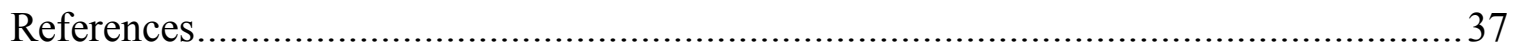

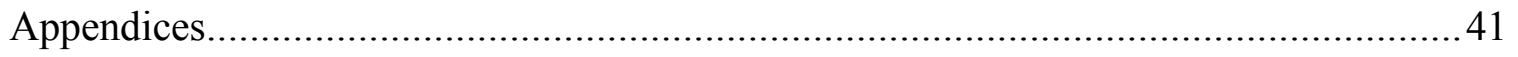


Barriers Faced by Nurses Caring for End-Of-Life Patients on a Medical Surgical Unit

\section{Background/Statement of the Problem}

The term end-of-life care describes the medical care and support that patients receive when faced with terminal illness. End of life care can take place in the months, weeks and days leading up to the patient's final heartbeat and last breath. End of life care can take place in many different settings such as homes, hospice centers and hospitals. Palliative care and hospice care are forms of end-of-life care that patients receive to control symptoms and improve quality of life.

Providing end-of-life care in any setting can be challenging. End-of-life care is specialized care and can have its own separate set of challenges. The acute care setting provides unique challenges to providing high quality end of life care. Acute care hospital settings tend to have a different level of urgency than other settings. Patients that require admission to the hospital setting are ill and need to be monitored closely. Patients have different levels of acuity, which can change abruptly in the acute care setting. Nurses caring for terminally ill patients in the general medical setting describe challenges they encounter, such as lack of education and experience, lack of time to spend with patients and families, as well as the physical environment of these units (McCourt, Power, \& Glackin, 2013).

Many patients will spend their final days and die in the hospital. Nurses in the acute care setting spend more time in direct contact with patients at the bedside than any other healthcare provider (Iglesias, Pascual, \& Vallejo, 2013). Nursing care on a medical-surgical unit can be very challenging in the effort to provide quality care to a diverse group of patients. Patients many times have more than one chronic illness in 
addition to the acute illness they were hospitalized for, and when the prognosis changes, a palliative care consultation may be sought. A formal hospital-based palliative care team is a more recent trend in healthcare (Milligan, 2012). This multidisciplinary team may consist of physicians, nurses, chaplains and counselors that specialize in palliative care. Consultation of the palliative care team can provide helpful assistance to patients, families and the healthcare team alike. This specialized team is able to provide direction regarding symptom control for the patient, medication management and also emotional and spiritual support to the patients and their families (Milligan).

Nursing has an extremely important role in providing quality care to palliative care patients, whose condition is often unpredictable. In the hospital setting, nurses are the essential caregivers who coordinate and deliver safe patient care (Hendrich, Chow, Skierczynski, \& Lu, 2008). Patients and their families count on nursing to ensure that they are comfortable during their final days of life. Nurses commonly describe a lack of ability to devote enough time to the dying patient and their families (Peterson et al., 2010). The nurses' role in caring for the dying patient includes medicating patients, providing physical and emotional care, supporting the family and also communicating with the team.

The majority of research on hospital based end-of-life care examines issues in quality of care and examines the patient and family's perspective, as well as the medical provider's perspective. Some research on the perspective of medical-surgical bedside nurses is available in the literature; however, the majority of the studies have been conducted within critical or palliative care settings (Thompson, McClement, \& Daeninck, 2006). The critical care setting greatly differs from a medical-surgical unit and in this 
setting it is not uncommon for a nurse to care for one to two critical patients. Critical care is a demanding environment in which the goals most often are lifesaving and curative (McCourt et al., 2013). Nurses and other health care professionals agree that patients who are terminally ill would receive the best care in a palliative care unit or hospice type setting in which the staff have more knowledge and education regarding end-of-life-care (McCourt et al.). A large majority of hospitalized patients receive care and pass away on medical units, therefore it is important that barriers to providing high quality end-of-life-care in this setting are investigated (Thompson et al., 2006)

The purpose of this project was to explore barriers to providing high quality endof-life care experienced by nurses in a medical-surgical setting.

Next, the literature review will be presented. 


\section{Literature Review}

The databases searched include CINAHL, Pub Med, and Google Scholar from January of 1997 to 2017. Search terms included the terms end-of-life-care, end-of-life care in the acute care setting, end-of-life care in medical-surgical setting, end-of-life in acute care hospitals, palliative care and nursing barriers to end-of-life care. Articles searched were published in the English language only.

\section{Nursing Practice on Medical-Surgical Units}

Medical-surgical units are fast paced environments with a broad array of patients that have a diverse set of diagnoses and complex interventions (Thompson et al., 2006). Some patients have more than one chronic illness each requiring testing and treatment. Other patients require admission to the medical-surgical unit for care after surgery. In today's society, patients and their families are consumers who have choices as to where and how they receive their healthcare. The patient's perspective regarding the quality of healthcare they receive is an important aspect in regard to improving care (Ahmad \& Alasad, 2004). However, the nursing perspective on the care provided is as fundamental as the patients' perspective of the care. They are often viewed as the frontline caregivers and have a critical role in delivering high quality and safe patient care (Hendrich et al., 2008).

A study called the "Time and Motion Study" (Hendrich, et al., 2008) was done to evaluate and quantify how nurses on medical-surgical units spent their time. The study was conducted on medical-surgical units at 36 different hospitals within 17 health care systems. One unit per hospital was randomly selected from all eligible medical-surgical 
units to be included in the study. Nurses that met the eligibility criteria were invited to voluntarily join the study. These nurses participated in research protocols that were created with the intent of assessing how bedside nurses' time is spent. The study more specifically assessed how much time nurses spent on specific activities such as 'nursing practice, nonclinical activities and waste, and unit-related functions'. The study also examined the average distance nurses traveled during a typical shift. The third aspect of this study was to determine how the work environment physiologically impacted the nurses.

The findings of this study confirmed that nurses in the medical-surgical areas spend a large portion of their time on documentation, medication administration, and coordination of care, which significantly impacts the amount of time that the nurse is able to spend at the bedside with the patient. Even with other disciplines providing care, including case managers, pharmacists, physical therapists and physicians, it is the nursing care that encompasses all of these specialties and more at all times (Hendrich et al., 2008). The nurse's role in end-of-life care requires that nurses spend with their patients and their families during the end of life process.

\section{Care of the Dying in Hospitals}

Acute care hospitals are recognized for caring for and treating acutely ill patients and are less recognized as a setting where patients receive end-of-life care (Caswell, Pollock, Harwood, \& Porock, 2015). In 2010, there were approximately 2.5 million deaths in the United States (US) (Hall, Levant, \& DeFrances, 2013). Despite research indicating that most Americans prefer to die in their own homes, about one-third of these 
deaths occurred in the hospital setting (Hall et al.). These figures do not differentiate what circumstances were present such as end-of-life care versus unexpected death or death related to acute illness. One key finding from the National Hospital Discharge Survey (NHDS) was that those who passed away in the hospital setting spent an average of 7.9 days compared to the 4.8 days for all patients admitted to the hospital (Hall et al.). These findings suggest that hospital systems need to educate nurses and healthcare personnel on end-of-life care in order for patients and their families to have a high quality experience during this difficult time. As research has shown, dying in the hospital setting has become increasingly more common.

\section{Definitions and Distinctions: Hospice; Palliative Care; End of Life}

It is believed that hospice originated centuries ago. In the Middle Ages, religious orders opened their doors to travelers who needed rest, food and shelter. "Hospice" stood for rest and shelter (Meier \& Bowman, 2017). These types of institutions evolved into safe places for people who were sick and elderly or disabled that did not have any other place to go. The first official hospice opened in London in 1967 by Dame Cicely Saunders. Florence Wald opened the first hospice in the US in 1974 in Connecticut. At that time, Wald also created an extensive hospice curriculum for Yale School of Nursing (Meier \& Bowman, 2017). The theme that came from the pioneers of hospice was that the care of terminally ill did not end when there was no longer a treatment option for the patients' disease. Hospice care was a treatment option aimed at providing care with the intent of keeping patients comfortable and free of pain related to the illness. In order to qualify for hospice care, patients cannot be receiving curative treatments and must be within six months to end-of-life. Hospice was recognized by 
Medicare and added as a benefit for patients near the end-of-life. Once Medicare added hospice as a benefit, many private insurers added hospice care as a benefit, which led to a significant amount of growth in hospice in the United States (Meier \& Bowman).

The development of palliative care evolved in response to the need for patients who were terminally ill to receive the best quality care which included management of the signs and symptoms of the illness, communication with the healthcare team and support provided to patients and families (Perrin \& Kazanowski, 2015). The World Health organization (WHO) (2014) stated that palliative care is intended to support patients and families during the period of diagnosis to end-of-life. Palliative care is intended to encompass the care of the mind, body, and soul.

The first palliative programs began in the late 1980's (Meier \& Bowman, 2017). Palliative care was created for patients who chose to continue to receive treatments aimed at curing disease, unlike hospice where treatments aimed at curing diseases were discontinued. Palliative care was created for individuals that didn't meet the eligibility requirements of hospice and also for those who wanted to continue to receive treatment and still deserved supportive care for their illness. In the late 1990's, palliative care programs were becoming more available. Nursing was the first clinical discipline to offer a certification in the area of palliative care (Meier \& Bowman). As palliative care grew, hospitals realized that there was a positive impact on the bottom line when palliative care consults were provided. As of $2017,66 \%$ of all hospitals nationwide were found to offer palliative care services. Palliative care in the hospital setting has become less of an exception and has had a positive impact on quality of life for patients suffering from chronic diseases (Meier \& Bowman). 
Patients and their families should have choices about where they choose to die. End-of-life care can be provided in different settings such as hospitals, nursing homes, patients' homes and facilities that specialize in end-of-life care such as hospice centers. Patients are more familiar with hospice care and tend to view hospice and palliative care as the same. Nurses are many times the first caregivers to facilitate discussions regarding health care decisions. Palliative care furthers discussions related to decision making about patients' goals of care to improve quality of life (Rodriguez, Barnato, \& Arnold, 2007).

\section{Patients' Perspective on End-of-Life}

To improve end-of-life care, the perspective of patients needs to be understood. Singer, Martin, \& Kelner (1999) identified end-of-life care to be among one of the most prevalent issues faced in healthcare because everyone dies at some time. Singer et al. performed a qualitative study to identify and describe what elements in terms of quality were important to patients requiring end-of-life care. The study was conducted in Toronto, Ontario and had a total of 126 participants from three patient groups. The first group consisted of 48 dialysis patients, the second had 48 participants with human immunodeficiency virus infection and the third patient group included 38 patients that were residents of a long-term care center. The data were gathered by in-depth openended questions through face-to-face interviews. The participants' views regarding quality end-of-life care were identified and the data went through a coding process and were then clustered into domains. 
The authors identified five main domains of quality related to patients' perspectives on quality end-of-life care. The most frequently identified domain was pain and symptom management. Treating pain and management of other symptoms has improved with better access to palliative care, though it still remains a problem for many patients at the end-of-life (Singer et al., 1999). The skills and attitudes of healthcare workers in regard to pain and symptom control could benefit from further study (Singer et al.).

The second domain that respondents identified was the inappropriate prolongation of the dying process. Respondent's responses included comments such as "becoming a vegetable", "lingering" and "being kept alive by a machine. (Singer et al., 1999). The third domain identified was achieving a sense of control. Participants insisted that they were the ones who should be in control of their end-of-life decisions until they are no longer able and at that time, the healthcare proxy would assume control (Singer et al.). The fourth domain identified the participants' fears their dying would be a burden. More specifically, the burdens identified included provision of physical care, witnessing their death and forcing others to make decisions regarding life-sustaining treatment. Patients concerns in regard to burdening their family members involved many variables. Patients acknowledged concerns regarding physical, financial and emotional burdens they would not want their families to experience (Singer et al.). The fifth domain identified the importance of strengthening relationships with loved ones. Good communication regarding the difficult subject matter that death and dying brings to light is extremely important. If this understanding can be achieved, an even closer bond could be formed. Patients found that once difficult discussions took place, their relationships were 
strengthened. Nurse can be a great support to a patient during difficult discussions between patients and families. Nurses communicate and form bonds with their patients and patients often times rely on the nurse to help them communicate with their families (Singer et al.).

A study by Heyland (2006) used a cross-sectional survey design to assess patients' and their families' perspectives related to key elements of quality of end-of-life care. Face-to-face questionnaires were administered to older patients with chronic endstage medical conditions and advanced cancer. The study took place from November 2001 through June 2003 at a tertiary care teaching hospital in Canada. Nurses administered the questionnaire to 434 participants (Heyland).

The results revealed that the most important elements of end-of-life care that the patients identified included trust in the physician providing their care, avoidance of unwanted life-support, effective communication, continuity of care and life completion. The results were compared to the results of previous studies that evaluated quality end of life elements. The elements that were not identified in previous studies were the trust and confidence in physicians, adequacy of discharge planning, and the honesty of communication families received were noted to be extremely important (Heyland).

\section{End of Life Care from the Nursing Perspective}

The majority of research focusing on end-of-life care examines issues in quality of care from the patient and family's perspective as well as the perspective of physicians (Thompson, et al., 2006). Research examining the perspective of nurses has been 
studied; however, the majority of these studies takes place within critical or palliative care settings (Thompson et al.).

Thompson et al. (2006) conducted a study to better understand the nursing process involved in providing high quality end-of-life care. Grounded theory method was used to understand the nurses' experience when caring for end-of-life patients. The study consisted of 10 nurses and took place in Canada at two tertiary university-affiliated hospitals. Face-to-face interviews were conducted on acute medical wards, in addition to direct observation of nurses on the medical wards.

The findings of the study signified that nurses, while they were trying to provide quality end-of-life care to their patients on an acute medical ward, felt they were often being pulled in many different directions. This author described difficulties nurses face when caring for patients in this environment. "Nurses who work on medical units juggle multiple patient care responsibilities and, therefore have to prioritize where to expend their time and energy" (Thompson et al., 2006, p. 173). Prioritizing care can be difficult to achieve for nurses who are trying to do their best and provide quality care to all of their patients and families. Nurses described feeling frustrated and having moral distress when caring for patients that they knew were at the end-of-life and should be receiving palliative care (Thompson et al.). Moral distress occurred because the nurse knew what was ethically correct but was unable to pursue the appropriate care due to the patient, family and institutional constraints.

The study described the process of a "lane change". The term lane change described a shift from acute care aimed at curing the disease to actively pursuing and 
providing comfort care (Thompson et al., 2006). Nurses described the difficulties endured when they were unsuccessful in achieving a lane change, stating that this led to feelings of helplessness and frustration. Nurses also described difficulties in getting what was needed for patients to receive palliative care due to the physicians' lack of training in palliative care situations. The nurses also felt strongly that "being there" was an important part of providing quality care to end-of-life patients. One important factor that affected the ability of the nurse to "be there" was the complexity of the environment of the medical surgical unit where the nurse had various competing priorities. Nurses cared for multiple patients, all of whom have needs for medications, cardiac monitoring, procedures at the bedside and other nurse specific activities. In addition, the authors reported that nurses value the connections they make with patients and their families at the end-of-life (Thompson et al.).

\section{Barriers to End-of-Life Care in Critical Care}

In the hospital setting, nurses play an important role in the care patients and their families receive during the time leading up to death. Through advancements in treatment and technology, patients are able to live longer. In the critical care setting, nurses care for patients at the end-of-life on a daily basis (Beckstrand, Callister, \& Kirchoff, 2005). Beckstrand reported results of a study conducted regarding obstacles and supportive behaviors critical care nurses perceived when providing nursing care to end-of-life patients. The study utilized a survey that consisted of quantitative and qualitative questions, which measured the nurses' perceptions of the frequency and intensity of occurrence of obstacles and supportive behaviors critical care nurses faced when providing end-of-life care. The questionnaire was adapted from the National Survey of 
Critical Care Nurses Regarding End-of-Life Care that was previously administered in 1998. The questionnaire was sent to 1500 randomly selected members of the American Association of Critical-Care nurses (AACN).

Beckstrand et al. (2005) observed the highest scoring obstacle nurses reported was frequent telephone calls from patients' family members for updates on patients' condition instead of directing the call to the designated contact person. The second obstacle identified was family members' difficulty in understanding what the term 'lifesaving measures' really meant. The third obstacle nurses faced when caring for end-of-life patients involved physicians on the patients' care team having a divergent opinion in regard to the direction of the patients' care goal. The supportive behaviors nurses scored highest included: spending time teaching and discussing the dying process with the family; patients' family members being able to spend an adequate amount of time at the bedside after the patient had passed; and having a peaceful and dignified place for the family to say goodbye after the patient has passed.

The results of this study confirmed how many different roles the nurse provides when caring for an end-of-life patient. The nurse is always at the bedside caring for the patients and their families throughout the end-of-life process. Nurses provide care, emotional support and are continually teaching patients and their families. Though conducted in critical care, findings are relevant to medical-surgical units.

In a descriptive study by Iglesias et al. (2013), a questionnaire was used to identify aspects of care that nurses consider to be facilitators or barriers to providing good care to patients and their families in end-of-life experiences. The study was conducted 
from October to December 2010. A contact person at each participating hospital collected the data. The survey was given to staff nurses with at least 3 months of experience working in the Intensive Care setting.

The top four items that nurses identified as helping in providing end-of-life care included: the possibility to have a peaceful end of life scene, free of extraordinary measures, a private place for family to cry; allowing family members to have enough time to be alone with the patient after he/she has passed; and the family accepting that the patient is dying. The four highest rated items related to obstacles to care identified were: physicians being evasive with families; providing painful interventions to the patient; families not accepting when the patient had a poor prognosis; physicians not allowing patients to die from the disease process; and families requesting life-saving measures, contrary to the patients' requests. Another important finding of this study was that nurses did not feel adequately trained in end-of-life care. Approximately $48 \%$ of nurses that responded have not taken end-of-life courses. This suggests that nurses may benefit from continuing education that focuses on end-of-life care (Iglesias et al.).

\section{Barriers to Providing End-of-Life Care on Medical-Surgical Units}

Caring for dying patients is not an aspect of nursing that comes easy to all nurses. Nurses in different settings have different comfort levels as it pertains to end-of-life care. Nurses are not always exposed to caring for dying patients. Yin, Xia, Yi, \& Chia (2000) used a descriptive study to explore nurses' attitudes toward caring for end-of-life patients on a medical oncology ward compared to nurses providing care on a general surgical ward. The study utilized a questionnaire that contained questions regarding nurses' 
views on caring for dying patients and also included sociodemographic data. A ChiSquare/Fisher's Exact test was used to assess the association between demographic variables and the various level of nurses' views on caring for dying patients.

Yin et al. (2000) reported that nurses working on medical-surgical units were more likely to agree that they were frightened of caring for dying patients. Nurses working on medical-oncology wards dealt with end-of-life patients on a daily basis and were well versed in helping their patients who were facing death. The results did not show a statistically significant difference between the two nursing units as it applied to nurses having feelings of helplessness and feelings of being fulfilled when caring for endof-life patients. The findings showed that $58.3 \%$ of the nurses in the general surgical ward and $61.9 \%$ in the medical oncology ward felt helpless when caring for their patients. In terms of nurses feeling fulfilled when caring for patients, the nurses on the general surgical ward scored $35.7 \%$ and the nurses on the medical oncology ward scored at $42.9 \%$. The results showed that $73.3 \%$ of nurses working on the medical-oncology ward were found to feel exhausted when caring for end-of-life patients. The results showed that $54.8 \%$ of nurses working on the medical-surgical unit felt exhausted taking care of dying patients. The authors concluded that nurses' own attitudes towards death can play a role in caring for a dying patient. The authors also contended that nursing care of dying patients is inevitable and nurses from areas such as the medical-surgical unit in this study all benefit from a good understanding of palliative care and caring for the dying patient (Yin et al.).

A literature review by McCourt et al. (2013) was conducted to explore the nurses' experience when providing end-of-life care to patients in the acute care hospital setting. 
Nine studies were analyzed and evaluated until themes emerged. The six themes that were prevalent in the literature review included lack of end-of-life education and knowledge, lack of ability to spend time with patients, barriers arising in the culture of the health-care setting, communication barriers, symptom management and nurses' personal issues.

The literature review findings described the difficulties nurses' face when caring for end-of-life patients in the acute hospital setting. Of the nine reviewed studies, seven consistently described nurses reporting lack of education and knowledge as a significant obstacle to providing quality end-of-life care to patients and their families. The lack of education and knowledge nurses' described compounded by other barriers to effective communication that were present between nurses, patients' and their families make providing care in the hospital environment very difficult. These barriers reinforce the need for improvement in education and training for nurses. Providing education to nursing is the first step to improve the care patients receive in the hospital setting (McCourt et al.).

The literature review confirmed that there are many issues nurses face when caring for end-of-life patients in the acute hospital setting. The recommendations of this literature review included improving education and training for nurses regarding end-oflife care, changes to the culture of care to meet patients' needs and emotional support for nurses providing end-of-life care (McCourt et al.).

One critical element of nursing care necessary for patients at end-of-life is ensuring that symptoms associated with dying are managed appropriately. Howes (2015) 
conducted a study to explore how nurses perceived the administration of end-of-life medications in medical and surgical settings. The purpose of the study was to identify variables that impacted nurses' use of the end-of-life medications in this setting. A qualitative descriptive design was used for this study. Data were collected during group interviews of registered nurses from eight medical-surgical units within a large teaching hospital.

The findings identified four main variables that impacted nurses' use of end-oflife medications when caring for dying patients in the medical-surgical settings. The variables included perceived knowledge and skill, differing perspectives with family members pertaining to use of medication, educational needs to improve knowledge and skills, comfort using end-of-life medications and nurses' emotional experience of taking care of patients at the end of life, which can create a positive or negative outcome (Howes, 2015). Nurses reported that fear related to lack of knowledge and assessment skills was a significant barrier related to the use of medication for symptom management. The author concluded that education and mentorship would be valuable to ensure that nurses in the hospital setting felt comfortable with these important concepts (Howes).

The findings of a recent study described the nurses' experience in caring for patients throughout the dying process (Oliveira, Fothergill-Bourbonnais, McPherson, \& Vanderspank-Wright, 2016). The purpose of the study was to describe the experience of nurses regarding the care they provide to end-of-life patients in the medical-surgical setting and to identify factors that promote and prevent nurses from being able to provide quality care to the dying patient. The research focused on developing a better 
understanding of the factors that affect nursing care on medical-surgical units in order to improve care in challenging environments.

The study was conducted in an 1149-bed teaching hospital in Canada. The sample consisted of $10 \mathrm{RNs}$ from two hospital medical-surgical units who were selected by the researcher through purposive sampling. Interpretive phenomenology was the design used in this study. Van Manen's approach was used, which describes a persons' firsthand experience. Face-to-face interviews were conducted using broad open-ended questions and the interviewer used prompting questions to further explore and understand the nurses' experience on a deeper level.

Participants in this study compared caring for end-of-life patients in the medical unit environment to being stuck in a spider's web (Oliveira et al., 2016). The first and most common theme identified was caring in complexity. This theme describes the challenges in caring for patients with complex needs that can change at any moment. It also discussed the complex illnesses that were easily exacerbated and then later went into remission. Theme two was described as caught in a tangled web. Participants sometimes perceived being caught in the middle when patients, families and physicians had different expectations. Theme three was bearing witness to suffering which described the struggle nurses faced when they were up against barriers that they had no control over. One specific difficulty was watching the patients' suffering and being unable to alleviate it, especially when the patients' condition worsened and the plan still remained curative.

The fourth theme identified was weaving a way to get there: struggling through the process. This theme described the struggle nurses encounter when wanting to be 
included by physicians as part of the healthcare team and have their professional opinion heard. Nurses felt excluded from the development of the plan of care. Nurses believed that they had valuable knowledge of the patient's health and needs and were often not involved in family meetings. The fifth theme described the difficulties in providing physical and emotional comfort to the patient and family in the chaotic environment of a medical unit. The sixth theme described the importance of bonding and forming a relationship with the patient and the family. The needs of the family can be emotional, information seeking and spiritual. The nurse has an important role in caring for a patient at the end-of-life, which always includes the families involved. The seventh theme identified how nurses 'found their way' through the challenges of caring for end-of-life patients. Nurses felt that they could rely on their nurse colleagues and that they supported each other through the tangled web (Oliveira et al.).

The findings of this study confirmed that nurses have an essential role in the care of an end-of-life patient. The results described the importance of having supportive and high-functioning nursing teams as a critical factor in ensuring the complex needs of the patients and families are met along with the other demanding routines nurses are also responsible for (Oliveira et al.).

Quality care is important to patients and families, but also to the nurses who care for them. The viewpoint of nurses is a key element in identifying the barriers and implementing ways to improve care. The experiences of nurses are valuable and can improve end-of-life care in the acute hospital setting.

Next, the framework that was used to guide this study will be presented. 


\section{Theoretical Framework}

The theory of Nurse Moral Distress by Corley (2002) was used to guide the development and implementation of this project. Jameton (1984) first described moral distress as painful feelings that occur when, because of institutional constraints, the nurse cannot do what he or she perceives to be what is needed.

Moral distress is not a new concept to nursing. Nurses' encounter situations daily that may cause moral distress. The theory of Moral Distress takes into consideration the internal and external factors that contribute to the moral distress that nurses face. The internal factors consist of the psychological response nurses experience. The external factors take into consideration the work environment at hand. The theory focuses on eight moral concepts, which include moral commitment, moral sensitivity, moral autonomy, moral sense making, moral judgment, moral conflict, moral competency, and moral certainty (Corley, 2002).

Corley (2002) described moral commitment as abiding by moral values and identified that while nurses have a strong sense of moral value, but may lack moral competency, which can lead to higher levels of moral distress. Moral competency is identified as the ability to see the moral importance in a situation, and behave in a morally acceptable manor. Moral integrity is adhering to moral values and is linked to one's own self-respect while moral sensitivity reflects the ability to see the moral conflict at hand, and understand when there is a situation that may be unstable. Corley identified that nurses may be morally sensitive but lack moral competency, which puts them at higher risk for experiencing moral distress. Moral commitment is described as the 
willingness to face the risks after experiencing a moral situation. Moral competency stems from being able to see the moral issue at hand and use good judgment leading to morally appropriate behaviors. Corley noted that moral autonomy reflects the independence and responsibility nurses are given in regards to making choices in patient care, while moral certainty is comfortably and confidently feeling that something is morally right or wrong, acting on it while risking personal and professional self in the process.

Corley's theory of Nurse Moral Distress identifies key research topics to help foster an understanding of what experiences nurses face and how to address them (2002). Often times nurses encounter situations in which they know what is best for that patient but this can conflict with what is best for the organization, the physician, the family, or even other patients, or, at least arguably, for society as a whole (Corley). Nurses many times are uncomfortable taking care of end of life patients. Nurses are uncomfortable caring for these patients because they do not have the experience or training to deliver this specialized care. Providing this type of care requires time and experience that nurses working on medical-surgical units don't always have. Corley cited many common situations that lead to moral distress among nurses including: continued life support even though it is not in the best interest of the patient; inadequate conversations about end-oflife care between providers and patients and their families; inappropriate use of healthcare resources; inadequate staffing or lack of appropriate training for staff; inadequate pain relief for patients; and patients and their families being presented with false hope were some of the more common experiences. 
Moral distress faced by nurses can lead to burnout and decreased job satisfaction, which in turn can have an effect on the patient and the organization (Corley, 2002). Nurses face situations that can prevent them from not only doing what may be ethically appropriate but also face situations that do not allow them to provide high quality care due to the demanding environment. Nurses are an important part of the healthcare team and if they experience moral distress, it can have a negative effect on patients and their families. Patients, fellow nursing staff, and the organization all will suffer when nurses are burdened with morally distressing situations. The understanding of nurses' viewpoints and opinions can greatly help improve care that patients receive. Nursing is always changing to improve safety and care. Identifying the issues that lead nurses to face moral distress will only help to improve nursing care.

Next, the methodology will be presented. 


\section{Methods}

\section{Purpose}

The purpose of this study was to determine what barriers registered nurses (RNs) faced while caring for end-of-life patients on a medical-surgical unit in the hospital setting.

\section{Research Question}

The research question was: What are the common barriers RNs face when caring for end-of-life patients on a medical surgical unit?

\section{Design}

The project utilized a survey design.

\section{Sample and Site}

The participants were RNs working on two medical surgical-2 (M/S 2) units at L\&M Westerly Hospital. L\&M Westerly Hospital is a 70-bed community hospital located in Westerly, Rhode Island. The two medical-surgical units are 32 bed units with telemetry monitoring. The potential sample size was approximately 20 nurses.

Participants needed to meet the following criteria: RN employed by the hospital and currently working on one of the medical-surgical units for a minimum of three months. Three months was chosen due to the fact that many new RNs were working on these units and were oriented for a time frame of 6-8 weeks. Three months would allow for orientation to have been completed and for the nurses to have cared for an end-of-life patient independently. 


\section{Procedure}

The first point of contact for this project was the Chief Nursing Officer (CNO). Written permission was given by the $\mathrm{CNO}$ for this project to be conducted (Appendix A). The nurse manager of the units also approved this project. This research was revewed by the RIC IRB and was determined to be exempt.

The nursing staff were informed of the project being conducted by an IRB approved email and also informational posters displayed in the break room. The surveys were made available to RNs in the conference room on each unit from April 6 through April 14, 2018. An IRB approved informational letter was provided with the survey explaining the aim of the project and ensuring anonymity and confidentiality of the responses. A secured box was available for staff to place completed surveys in.

A raffle ticket was attached to each survey. A separate box was available for nurses to drop the ticket in once they had completed their survey. Once the survey period was over, two raffle tickets were randomly pulled from the box. The holder of the first ticket chosen received a $\$ 20$. gift certificate to the local uniform store and the second received a gift card to the local coffee shop.

\section{Measurement}

The End of Life Care Clinical Nurse Survey (Ferrell, Virani, \& Grant, 1999) was developed by the City of Hope researchers to assess the needs of clinical nurses in endof-life care. A modified version of this survey was used to identify the common barriers faced by nurses providing end-of life-care. The original survey contained a total of 30 
questions. Questions were removed that addressed issues that were not relevant to this project such as questions that asked if nurses supported euthanasia or assisted suicide.

The revised survey consisted of a total of six questions that were selected because they focused on nursing care and were aimed at identifying barriers RNs face when caring for end-of-life patients on a medical-surgical unit (Appendix B). The first question confirmed that the participant cared for end-of-life patients in their current role. If the participant responded no to this question, the data were not included in the analysis. The second question addressed perceived effectiveness of six aspects of end-of-life care such as pain management, psychological support, spiritual needs, and grief/bereavement support. The participant was asked to respond by circling 1 for 'not at all effective', 2 for 'somewhat effective', or 3 for 'very effective'. All responses were scored as percentages per category. The third question addressed 12 barriers to providing quality care, to which the participant could circle 1 for 'not a barrier', ' 2 for somewhat a barrier' or 3 for 'severe barrier'. The fourth question was qualitative and asked participants to identify what they felt was the greatest barrier to providing high quality care that they were faced with in their practice. The fifth and sixth questions included demographics such as level of education and how long the participant had been an RN. A pilot of the revised measure was conducted with two to three nurses who had worked on the medical surgical units within the hospital within the last year.

\section{Data Analysis}

Descriptive statistics were used to analyze the survey. Open-ended questions were analyzed to identify major themes reported by the nurses. Data once analyzed were 
stored on a thumb drive in a locked file cabinet that only the primary investigator had access to and will be stored for two years.

Next, the results will be presented. 


\section{Results}

A total of 15 nurses out of 20 eligible nurses (74\%) from Westerly Hospital participated in the survey. The nurses who responded became licensed as RNs between 1976 and 2017. Of the 15, seven (58\%) held an Associate's degree, four (33\%) held a Bachelor's degree, and one (12\%) held a Master's degree. Three subjects did not respond to this question. Nurses' perceived effectiveness of six aspects of end-of-life care are illustrated in Table 1 below.

Table 1

Effectiveness of end-of-life care aspects on M/S 2 and M/S 3

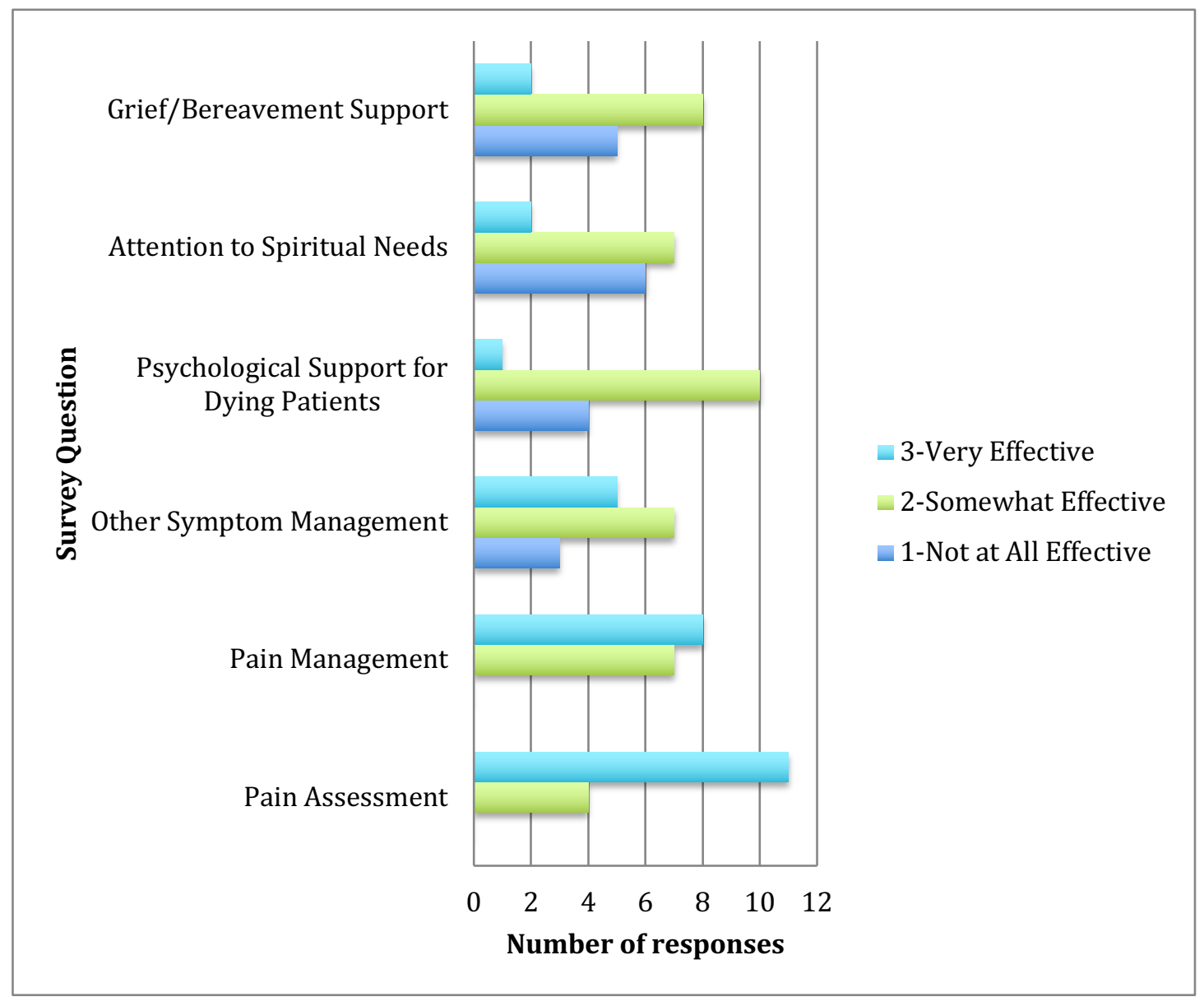


The survey responses demonstrated that pain assessment $(n=11 ; 73 \%)$, pain management $(n=8 ; 53 \%)$, and other symptom management $(n=5 ; 34 \%)$ were areas that the nurses felt was very effective on their unit. Only four nurses $(n=4 ; 27 \%)$ responded that pain assessment was somewhat effective. The majority of nurses felt that psychological support was somewhat effective $(n=10 ; 67 \%)$. Grief and bereavement support was felt to be somewhat effective by $53 \%$ of the nurses. Of the remaining responses, only 2 nurses $(n=2 ; 13 \%)$ felt that grief and bereavement support was very effective and 5 nurses $(n=5 ; 33 \%)$ felt that this was not at all effective. Attention to spiritual needs of patients was identified as being somewhat effective ( $n=7 ; 47 \%)$ and not at all effective $(n=6 ; 40 \%)$. The remaining $(n=2 ; 13 \%)$ responses demonstrated that nurses felt that attention to spiritual needs was very effective.

The third question identified 12 barriers to providing quality care to which the participant could circle 1 for 'not a barrier', '2 for somewhat a barrier' or 3 for 'severe barrier'. The results are shown in Table 2 on the next page. 
Table 2

Barriers to providing good end-of-life care on M/S 2 and M/S 3

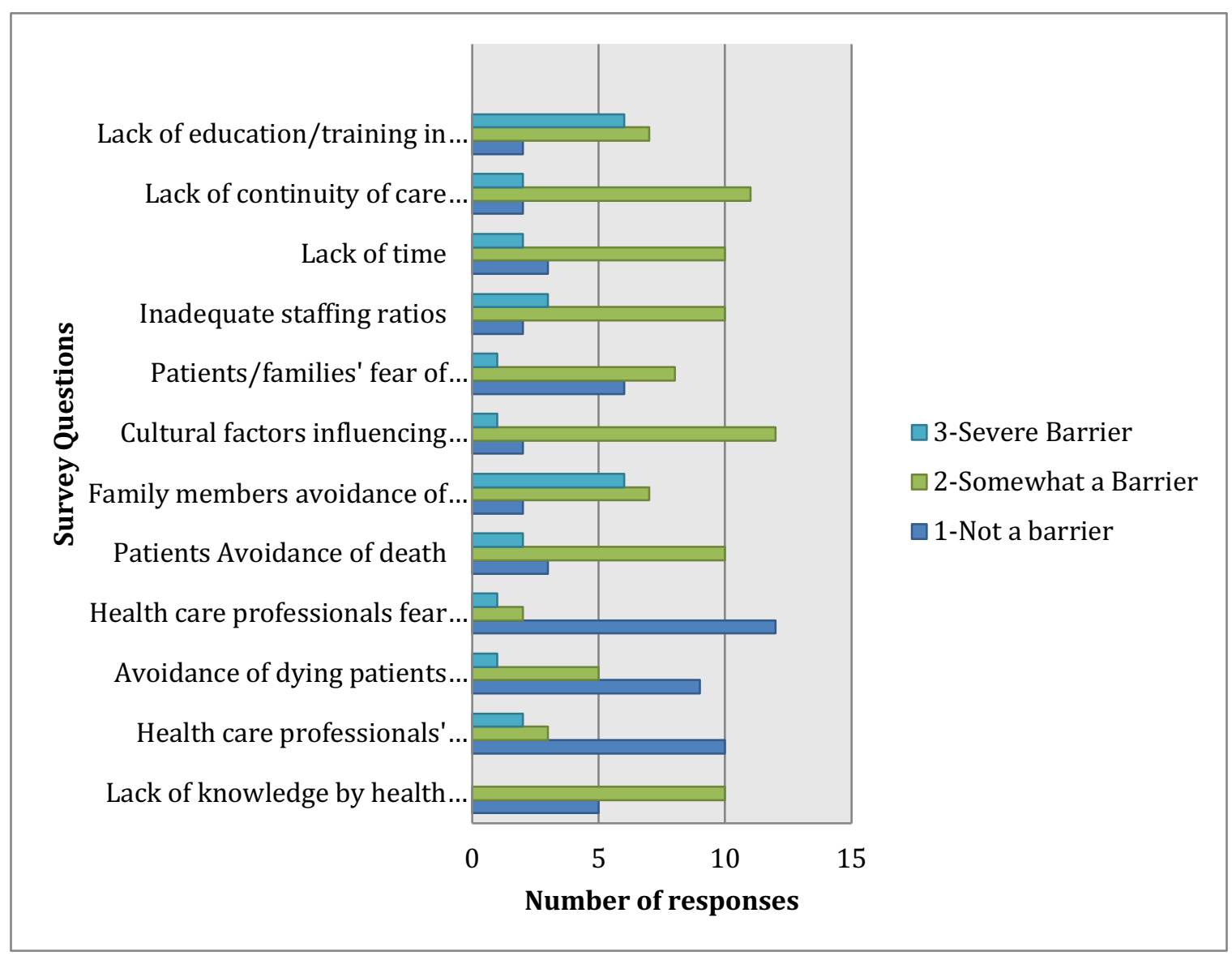

The results demonstrated that many of the factors posed somewhat of a barrier to providing good end-of-life care to the patients. The barriers that the nurses identified to be somewhat of a barrier and severe barrier to providing good quality care were family members' avoidance of death $(n=13 ; 87 \%)$ and lack of education and training in end-oflife care $(n=13 ; 87 \%)$. Cultural factors were identified by $(n=12 ; 80 \%)$ the nurses as somewhat a barrier to providing good quality care. Lack of continuity of care was identified ( $\mathrm{n}=11 ; 73 \%$ ) as somewhat of a barrier to providing good quality care. Nurses 
$(\mathrm{n}=10 ; 67 \%)$ did not feel that health care professionals personal discomfort with death was a barrier to providing good quality care. Health care professionals' fear of causing addiction by administering pain medications $(n=10 ; 67 \%)$ was also identified to not be a barrier.

The responses to the open-ended question that assessed what the greatest barriers nurses felt that they encountered when caring for end-of-life patients had common themes. The most common responses were lack of time to spend with patients and families $(n=4 ; 36 \%)$ and lack of education and training $(n=5 ; 45 \%)$. One nurse wrote "Being able to spend time with the patient and their families is difficult while managing the rest of your patient assignment". Another nurse stated "There is never enough time to spend with families".

Another common response included that frequent questions from the families could become very time consuming for the nurses when providing care. Two nurses noted unrealistic expectations from families were a barrier they encountered, especially when the families were "not in agreement about patients' wishes". One participant summarized what two other nurses identified, "Lack of education on medication administration for nurses who have not really dealt with end-of-life patients can lead to an uncomfortable patient and requires a lot of 'catch up' on the next shift to make the patient comfortable". Two responses $(n=2 ; 18 \%)$ identified that there was a lack of communication between the hospice $\mathrm{RN}$ and the physician overseeing patients' care when patients were receiving inpatient hospice care.

Next, the summary and conclusions will be presented. 


\section{Summary and Conclusions}

In the critical care setting, nurses care for patients at the end-of-life on a daily basis (Beckstrand et al., 2005). Research investigating barriers to providing end-of-life care in the critical care setting has received much more attention than end-of-life care in the medical-surgical setting. Medical-surgical units are fast paced environments with a broad array of patients that have a diverse set of diagnoses and complex interventions (Thompson et al., 2006). These environments present a diverse set of challenges for nurses when caring for end-of-life patients. One approach to determining the barriers to providing high quality care to end-of-life patients in this setting is to obtain feedback from nurses providing the care.

The purpose of this study was to determine what barriers registered nurses (RNs) faced while caring for end-of-life patients on a medical-surgical unit in the hospital setting. The theory of Nurse Moral Distress by Corley (2002) was used to guide the implementation of this project. Corley's (2002) theory of moral distress describes what can happen when nurses are unable or feel unable to advocate or do what is felt to be best for their patients. Often times nurses encounter situations in which they know what is best for that patient but this can conflict with what is best for the organization, the physician, the family, or even other patients, or, at least arguably, for society as a whole (Corley).

Written permission was given by the $\mathrm{CNO}$ for this project to be conducted and the nurse manager of the units also approved this project. The RIC IRB determined the project to be exempt. The participants were RNs working on units medical surgical-2 (M/S 2) and medical surgical-3 (MS/3) at L\&M Westerly Hospital, a 70-bed community 
hospital located in Westerly, Rhode Island. M/S 2 and M/S 3 are 32 bed mixed medicalsurgical units with telemetry monitoring. A modified version of the End of Life Care Clinical Nurse Survey (Ferrell et al., 1999) was used to assess the barriers faced by nurses in the medical-surgical setting at L\&M Westerly Hospital.

The results of the survey reinforced that nurses in the medical-surgical setting are faced with barriers that make caring for end-of-life care patients challenging. Lack of time and lack of education/training were identified as barriers to providing good end-oflife care. Nurses described "managing your patient assignment is difficult" while caring for an end-of-life patient, especially because their "is never enough time". This was confirmed in the responses received from the open-ended questions. The barriers stated in the literature did align with the results of this survey. One main barrier that was addressed in multiple studies was improving education and training for nurses regarding end-of-life care. The other barrier that aligned with the results of this study was the lack of time to spend with patients that nurses described.

A limitation that may have impacted the results of the survey was lack of experience of the nursing staff. Many $(\mathrm{n}=8 ; 53 \%)$ nurses had less than five years of experience in this setting. There were also approximately eight newer nurses that were not be able to complete the survey because they had not yet cared for end-of-life patients in their new role. Another limitation that may have impacted the study was a required staff satisfaction survey that was distributed at the same time.

Overall, the results from the survey reinforced that lack of time and lack of training are important barriers to providing quality end-of-life care. It was clear from this 
study that nurses wanted to be able to spend more time with their patients and viewed not being able to do so as a barrier to providing the best care.

Next, recommendations and implications for advanced nursing practice will be presented. 


\section{Recommendations and Implications for Advanced Nursing Practice}

Advanced practice registered nurses (APRNs), especially in the acute care hospital setting, are uniquely qualified to assist nurses to provide the highest quality care to end-of-life patients. As well as being a valuable resource for nursing colleagues, APRNs can advocate for improvements in end-of-life care in the medical-surgical setting. Nurses are required to have advanced training to care for certain patient populations such as cardiac patients requiring telemetry and are trained regarding cardiac medications and cardiac monitoring; however nurses are not typically provided any special education to care for end-of-life patients. The APRN is prepared to educate and could develop and conduct informational teaching sessions for the nurses regarding various end-of-life topics, including symptom management. Education on caring for end-of-life patients should be incorporated into the orientation period for new nurses and to on-going education for seasoned, practicing nurses.

Research by WHO (2014) stated that providing palliative should be considered an ethical duty for health professionals. Certification is available for RNs and APRNs through End-of-Life Nursing Education Consortium (ELNEC) ("American Association of Colleges of Nursing (AACN) End-of-Life-Care (ELNEC)", 2018). This certification focuses on core areas in end-of-life/palliative care to improve RNs and APRNs knowledge and skill to provide this specialized care and improve the experience patients and families face during this difficult time.

Advanced practice nurses are an important part of the interdisciplinary team in the medical-surgical setting. An interdisciplinary team consisting of physicians, social work, 
case managers, and nurses and APRNs specializing in palliative or hospice care would be beneficial in assisting nursing staff to provide the highest quality care. A collaborative approach is beneficial to the providers, patients, and families and could also be extremely beneficial to the nurses caring for these patients. Collaboration with other specialties could provide support and education to the nursing staff, with the goal of improving the quality of care.

The APRN possesses the ability influence change in the institutions in which they practice by creating protocols and policies to help guide the delivery of high quality care to end-of-life patients. This could be accomplished by creating a protocol to assist nurses to identify and treat common signs and symptoms experienced by end-of-life patients. Integrating this protocol and educating the nursing staff could help improve the quality of care patients receive at the end of their lives. A policy outlining the process for providing care and support for patients and families who are facing end-of-life would also be a useful tool. Such a policy should include key end-of-life information such as code status and comfort measures. Advance practice nurses are also able to impact policy by participating in professional organizations at the state and national level.

The APRN uses current research and evidence-based care to make improvements in practice. Advance practice nurses have a wealth of experience and knowledge, which they can use in leadership roles to advocate for safe patient care and high quality outcomes. Further research could help identify best practice strategies for designing and delivering appropriate educational needs for nurses caring for end-of-life patients. Research to identify nursing educational needs more specifically, such as medications used in end-of-life care for symptom management, would assist in developing targeted 
strategies. It would also be useful to research which medications are most effective in end-of-life care for symptom management. Another area for research is the lack of time nurses describe when caring for end-of-life patients in the medical-surgical setting. Observational studies to examine contributors to this perceived lack of time would be useful.

Providing high quality care to end-of-life patients in the medical-surgical setting requires collaboration between many different specialties. Nurse practitioners are in an ideal position to assess the needs of this patient population and collaborate with other members of the healthcare team to ensure that patients are receiving the high quality care they deserve. 


\section{References}

Ahmad, M. \& Alasad, J. (2004). Predictors of patients' experiences of nursing care in medical-surgical wards. International Journal of Nursing Practice, 10(5), 235241. http://dx.doi.org/10.1111/j.1440-172x.2004.00484.x

American Association of Colleges of Nursing (AACN). End-of-Life-Care (ELNEC). (2018). Retrieved from http://www.aacnnursing.org/ELNEC

Beckstrand, R., Clark Callister, L., \& Kirchoff, K. (2005). Providing a "Good Death": Critical care nurses.' suggestions for improving end-of-life care. American Journal Of Critical Care, 15(1), 38-45.

Caswell, G., Pollock, K., Harwood, R., \& Porock, D. (2015). Communication between family careers and health professionals about end-of-life care for older people in the acute hospital setting: A qualitative study. BMC Palliative Care, 14(1). http://dx.doi.org/10.1186/s12904-015-0032-0

Corley, M. (2002). Nurse moral distress: A proposed theory and research agenda. Nursing Ethics, 9(6), 636-650. http://dx.doi.org/10.1191/0969733002ne557oa

Ferrell, B. R., Virani, R., \& Grant, M. (1999). Analysis of end-of-life content in nursing textbooks. Oncology Nursing Forum, 26(5), 869-876.

Hall, M., Levant, S., \& DeFrances, C. (2013). Trends in Inpatient Hospital Deaths: National Hospital Discharge Survey, 2000-2010. U.S. Department Of Health and Human Services. Retrieved 10 August 2017, from http://us department of health and human services 
Hendrich, A., Chow, M., Skierczynski, B., \& Lu, Z. (2008). A 36-hospital time and motion study: how do medical-surgical nurses spend their time? The Permanente Journal, 25-34. http://dx.doi.org/10.7812/tpp/08-021

Heyland, D. (2006). What matters most in end-of-life care: perceptions of seriously ill patients and their family members. Canadian Medical Association Journal, 174(5), 627-633. http://dx.doi.org/10.1503/cmaj.050626

Howes, J. (2015). Nurses' perceptions of medication use at the end of life in an acute care setting. Journal of Hospice \& Palliative Nursing, 17(6), 508-516. http://dx.doi.org/10.1097/njh.0000000000000192

Iglesias, M., Pascual, C., \& Vallejo, R. (2013). Obstacles and helpful behaviors in providing end-of-life care to dying patients in intensive care units. http://dx.doi.org/10.1097/dcc.0b013e3182808429

Jameton, A. (1984) Nursing practice: The ethical issue. Englewood Cliffs, NJ: Prentice Hall.

McCourt, R., Power, J., \& Glackin, M. (2013). General nurses' experiences of end-of-life care in the acute hospital setting: a literature review. International Journal of Palliative Nursing, 19(10), 510-516. http://dx.doi.org/10.12968/ijpn.2013.19.10.510

Meier, D. \& Bowman, B. (2017). The Changing Landscape of Palliative Care. Journal of The American Society On Aging, 41(1), 74-80.

Milligan, S. (2012). Optimizing palliative and end of life care in hospital. Nursing Standard, 26(41), 48-56. http://dx.doi.org/10.7748/ns2012.06.26.41.48.c9157 
Oliveira, I., Fothergill-Bourbonnais, F., McPherson, C., \& Vanderspank-Wright, B. (2016). Battling a tangled web: the lived experience of nurses providing end-oflife care on an acute medical unit. Research and Theory for Nursing Practice, 30(4), 353-378. http://dx.doi.org/10.1891/1541-6577.30.4.353

Perrin, K. \& Kazanowski, M. Barriers to palliative care consultation. Crit Care Nurse 2015; 35:44-52

Peterson, J., Johnson, M., Halvorsen, B., Apmann, L., Chang, P., \& Kershek, S. et al. (2010). What is so stressful about caring for a dying patient? A qualitative study of nurses' experiences. International Journal of Palliative Nursing, 16(4), 181187. http://dx.doi.org/10.12968/ijpn.2010.16.4.47784

Rodriguez, K., Barnato, A., \& Arnold, R. (2007). Perceptions and utilization of palliative care services in acute care hospitals. Journal of Palliative Medicine, 10(1), 99110. http://dx.doi.org/10.1089/jpm.2006.0155

Ruland, C. \& Moore, S. (1998). Theory construction based on standards of care: a proposed theory of the peaceful end of life. Nursing Outlook, 46(4), 169-175. http://dx.doi.org/10.1016/s0029-6554(98)90069-0

Singer, P., Martin, D., \& Kelner, M. (1999). Quality end-of-life care. JAMA, 281(2), 163. http://dx.doi.org/10.1001/jama.281.2.163

Thompson, G., McClement, S., \& Daeninck, P. (2006). Nurses' perceptions of quality end-of-life care on an acute medical ward. Journal of Advanced Nursing, 53(2), 169-177. http://dx.doi.org/10.1111/j.1365-2648.2006.03712.x 
World Health Organization (2017). Palliative care. Retrieved 20 May 2017, from http://www.who.int/mediacentre/factsheets/fs402/en/

Yin, O., Xia, Z., Yi, X., \& Chia Chia, D. (2000). Nurses' perceptions towards caring for dying patients in oncology ward and general surgical ward. Singapore Nursing Journal. 


\section{Appendix A}

To: Rhode Island College Nursing (MSN) Department

Beth Dufour has permission to survey Registered Nurses at L\&M Westerly Hospital regarding nursing barriers to care in the medical-surgical setting. I understand that this will take place sometime during the fall/early winter.

Thank You, Kerin A. Da cruz, isn, Rn, NE-BC Chief Administrative Officer Interim Chief Nursing Office

Westerly Hospital 
Appendix B

\section{Nursing Survey Regarding End-of-life Care on Medical-Surgical Units}

1. Do you care for dying patients in your current role? Yes No (If no please stop here)

2.) How effective are the following aspects of EOL (End-of-life) care on your unit? (Please Circle your number choice using $1=$ Not at all effective, $2=$ Somewhat effective or $3=$ very effective)

Pain Assessment:

123

Pain Management:

$\begin{array}{lll}1 & 2 & 3\end{array}$

Other symptom management:

123

Psychological support for dying patients:

1

23

Attention to spiritual needs:

1

2

3 


\section{Grief/bereavement support:}
1
2
3

3.) How much of a barrier are the following factors to providing good end-of-life care in your setting? Please circle the appropriate number using $1=$ not a barrier, $2=$ somewhat a barrier or $3=$ severe barrier

Lack of knowledge by health care professionals:
1
2
3

Health care professionals' personal discomfort with death:
1
2
3

Avoidance of dying patients by health care professionals:
1
2
3

Health care professionals fear of causing addiction by administering of causing addiction by administering pain medications:
1
2
3

Patients' avoidance of death: 
Family members' avoidance

of death:

1

2

3

Cultural factors influencing end-of-life care:

123

Patients'/families' fear of addiction:

$\begin{array}{lll}1 & 2 & 3\end{array}$

Inadequate Staffing ratios:

$\begin{array}{lll}1 & 2 & 3\end{array}$

Lack of Time:

$\begin{array}{lll}1 & 2 & 3\end{array}$

Lack of continuity of care across settings:

$\begin{array}{lll}1 & 2 & 3\end{array}$

Lack of Education/training in End-of-life Care:

$\begin{array}{lll}1 & 2 & 3\end{array}$ 
4.) What is the greatest barrier to providing quality care you face as a nurse when caring for end-of-life patients?

5.) What year were you licensed as a registered nurse?

6.) What is the highest level of education you've completed?

_ Diploma __ ADN _ _ BSN/BS/BA _ _ MSN/MS/MA _ _ PhD/EdD/DNS

Adapted from: Ferrell, B. R., Virani, R., \& Grant, M. (1999). Analysis of end-of-life content in nursing textbooks. Oncology Nursing Forum, 26(5), 869-876. 